\title{
Electroencephalographic spectral analysis: detection of cortical activity changes in sleep apnoea patients
}

\author{
K. Dingli*, T. Assimakopoulos\#, I. Fietze ${ }^{\Uparrow}$, C. Witt ${ }^{\ddagger}$, P.K. Wraith*, N.J. Douglas*
}

Electroencephalographic spectral analysis: detection of cortical activity changes in sleep apnoea patients. K. Dingli, T. Assimakopoulos, I. Fietze, C. Witt, P.K. Wraith, N.J. Douglas. C ERS Journals Ltd 2002.

ABSTRACT: There are no visible electroencephalographic (EEG) changes at the termination of some apnoeas and hypopnoeas. This study tests the hypothesis that cortical activity fluctuates at apnoea/hypopnoea termination, despite the lack of visible changes.

To detect these changes, EEG spectral analysis was performed and centred around the end of apnoeas/hypopnoeas in $\mathbf{1 5}$ sleepy patients. Ten second windows were applied and comparisons were conducted between the normalised power of the same frequency bands before and after termination of each apnoea/hypopnoea. Comparisons were performed within patients between apnoeas/hypopnoeas and periods of undisturbed sleep as well as between patients and healthy subjects during sleep.

Normalised theta power $(4-8 \mathrm{~Hz})$ decreased significantly at apnoea/hypopnoea termination. No significant changes were found between consecutive periods of undisturbed sleep across the 15 patients. During nonrapid eye movement sleep, changes were detected irrespective of arousal visibility. During rapid eye movement sleep, nonarousal apnoeas/hypopnoeas were not accompanied by any significant spectral power changes. Theta power was significantly lower across patients compared to healthy subjects $(\mathrm{p}=0.03)$ and was correlated to the apnoea/hypopnoea index $(\mathrm{rho}=0.6, p=0.008)$.

The authors conclude that electroencephalographic spectral analysis improves detection of changes at apnoea/hypopnoea termination. Further validation is needed to determine whether it improves correlation between nocturnal measures and daytime symptoms. Eur Respir J 2002; 20: 1246-1253.
*Respiratory Medicine Unit, University of Edinburgh and "Dynesys Ltd, Edinburgh, UK. Respiratory Medicine, Charite, Berlin, Germany.

Correspondence: N.J. Douglas

Dept of Medicine

Royal Infirmary

Edinburgh

EH3 9YW

UK

Fax: 441315363255

E-mail: n.j.douglas@ed.ac.uk

Keywords: Electroencephalography power spectrum

sleep apnoea syndrome

Received: October 222001

Accepted after revision: July 122002

K. Dingli was supported by the European Respiratory Society in the form of a research fellowship.
Cortical arousals induced by apnoeas and hypopnoeas during sleep are believed to be the primary cause of sleep fragmentation, daytime sleepiness, impaired cognitive function and concentration in obstructive sleep apnoea/hypopnoea syndrome (OSAHS) patients. However, previous studies have found a weak correlation between severity of clinical symptoms or objective sleepiness and polygraphical findings, in terms of apnoeas, hypopnoeas and cortical arousals [1-3].

Approximately $28 \%$ of apnoeas and hypopnoeas are not terminated by visible cortical arousals [4]. This could be due to the limitations of visual scoring to detect all arousals. This suggestion is supported by the findings of MARTIN et al. [5] who showed that daytime sleepiness was increased in healthy subjects by applying stimuli which caused autonomic changes but no visible changes in cortical activity. The same study showed a significant increase in alpha band power when tones induced visible arousals and also when they did not.

It has previously been suggested that differences in arousability are associated to the depth of sleep [6]. Nonconventional techniques of electroencephalographic (EEG) analysis such as neural networks [7, 8] and power spectral analysis [6,9] succeeded in improving detection of cortical activity changes during apnoeas/ hypopnoeas and at their termination, irrespective of arousal visibility or sleep stage during which they occurred.

EEG power spectral analysis during apnoeas/hypopnoeas [9] and during periods of increased inspiratory effort, detected through oesophageal manometry [6], showed increase in delta power towards the end of these events. The degree of increase varied in parallel to the sleep cycles.

The present study tests the hypothesis that detection of cortical activity changes at apnoeas/hypopnoeas termination may be improved through the application of spectral analysis. The power spectrum of the EEG signal has been analysed in order to improve the detection of nonvisible signal changes related to apnoea/hypopnoea termination. The detected sleeprelated spectral power changes in OSAHS patients were compared to healthy subjects.

\section{Methods}

\section{Subjects}

The polysomnograms of 15 subjects suspected of having OSAHS (14 males, aged $51 \pm 9$ yrs (mean \pm SD), 
body mass index (BMI) $29 \pm 2 \mathrm{~kg} \cdot \mathrm{m}^{-2}$ ) and seven healthy, asymptomatic subjects (four males, aged $50 \pm 10$ yrs, BMI $28 \pm 2 \mathrm{~kg} \cdot \mathrm{m}^{-2}$ ) in whom OSAHS had previously been excluded, were analysed. The patients' main symptom was daytime sleepiness. Autonomic, endocrine, cardiopulmonary dysfunction, periodic limb movement disorder, other neurological disorders or intake of medication which affected sleep were excluded. The study was approved by the institutional ethics committee.

\section{Polysomnography}

Polysomnography was performed using a computerised recording system consisting of: 1) two central (C3A2, C4A1) and two occipital (O1A2, O2A1) EEG tracings, two outer canthi electrodes (LEOG, REOG) and a submental electromyography (EMG) electrode; 2) tibial EMG; 3) three-lead electrocardiogram; 4) oronasal airflow detection through a thermistor sensor, thoracoabdominal movement detection through two piezoelectric belts, a digital microphone and a pulse oximeter.

Recordings were carried out between 22:00-7:00 h. They did not include a continuous report on the supine body position.

The used polysomnography system had a digital hardware Butterworth filter of third order with cut-off frequencies set at 0.6 and $50 \mathrm{~Hz}$ with $12 \mathrm{~dB}$ per octave.

\section{Scoring/definitions}

Scoring was performed by a single observer, blinded to subject. Approximately $7 \%$ of the data were excluded due to movement or EEG artefacts. Sleep was scored on the C4A1 and C3A2 tracings according to the RECHTSCHAFFEN and KALES [10] criteria.

Arousal scoring was based on the definition of the American Sleep Disorders Association (ASDA), modified as to the duration threshold which was set at $1 \mathrm{~s}$ rather than $3 \mathrm{~s}$ [11]. Arousal scoring ranged from 1-15 s, irrespective of sleep re-occurrence or prolonged awakening subsequent to the arousal; hence, RECHTSCHAFFEN and KALES [10] awakenings were also included in the scoring. The number of arousals adjacent to apnoeas/hypopnoeas per hour of sleep, was termed the respiratory arousal index (RAI).

Apnoeas were defined as a cessation of the oronasal airflow. Hypopnoeas were defined as a clear amplitude reduction of the airflow of $>50 \%$ compared to a $10 \mathrm{~s}$ peak amplitude during the preceding $2 \mathrm{~min}$, lasting $\geqslant 10 \mathrm{~s}$ and associated with either an oxygen desaturation of $\geqslant 3 \%$ or an arousal [12].

Arousals and respiratory events were scored during two separate sessions. To assess reproducibility of scoring outcomes, the 15 polysomnograms were scored twice by the same researcher. In these intrarater analyses the researcher was blind to the patient details and the results of the comparison studies. Intrarater comparisons were made 12 months after the initial study was scored. Variability was calculated as the difference between the two scores in per cent.

\section{Post acquisition analysis}

The power spectrum of the C4A1 tracing [13, 14] was analysed and the power spectral density (PSD) curve was generated, i.e. power distribution as a function of the EEG's constituent frequencies. For the PSD calculation, the nonparametric Fast Fourier Transform (FFT) algorithm was applied, using the technique as described by Welch et al. [15]. The normalised power values, i.e. the absolute band power value as a fraction of the total power were calculated for the following frequency bands: 1) Delta frequency band: $1-4 \mathrm{~Hz}$; 2) theta frequency band: $4-8 \mathrm{~Hz} ; 3$ ) alpha frequency band: $8-12 \mathrm{~Hz}$; 4) sigma frequency band: $12-16 \mathrm{~Hz}$.

Off-line analysis was performed using a software package (Dynesys Ltd, Edinburgh, UK). The DC-free EEG signal (sampled with $100 \mathrm{~Hz}$ ) was filtered with a Finite Impulse Response (FIR) low-pass filter and a cut-off frequency at $25 \mathrm{~Hz}$ [16]. For the calculation of the power density a 128 bin Hamming window with 64 points overlap and a resolution of $0.015 \mathrm{~Hz}$ was applied. For the delta, theta, alpha and sigma frequency bands the area under the spectral curve was calculated using a fifth-order Gaussian integration method, yielding power expressed in microVolt ${ }^{2}$ [17] (fig. 1).

The periods analysed centred around the end of apnoeas/hypopnoeas, identified as increase/recovery of the airflow amplitude. To assess power changes at apnoea/hypopnoea termination, comparisons were conducted between the synonymous frequency bands $10 \mathrm{~s}$ before versus $10 \mathrm{~s}$ after the end of each apnoea/ hypopnoea.

The time windows were chosen to maintain signal stationarity within the applied window, as required for the application of the FFT algorithm. The $10 \mathrm{~s}$ window would in most cases avoid overlap between postapnoeic/-hypopnoeic intervals and the following early apnoeic/hypopnoeic periods. The further aim of the chosen windows was to maximise the signal-tonoise ratio by a compromise between occupying as much of the window as possible with potential arousals while at the same time allowing a reasonably wide window to cut down on random variance in the EEG signal (fig. 2).

To determine whether the found power changes were caused by apnoeas/hypopnoeas, the authors compared the outcomes to periods of undisturbed sleep of the same duration in rapid eye movement (REM) and nonrapid eye movement (NREM) sleep, during which no apnoeas/hypopnoeas occurred. These periods were not necessarily free of snoring.

To assess the significance of the EEG power changes found across OSAHS patients these were compared to the EEG power spectrum of healthy subjects.

\section{Statistical analysis}

For the before versus after apnoea/hypopnoea termination-related comparisons as well as for the comparison between consecutive periods of undisturbed 

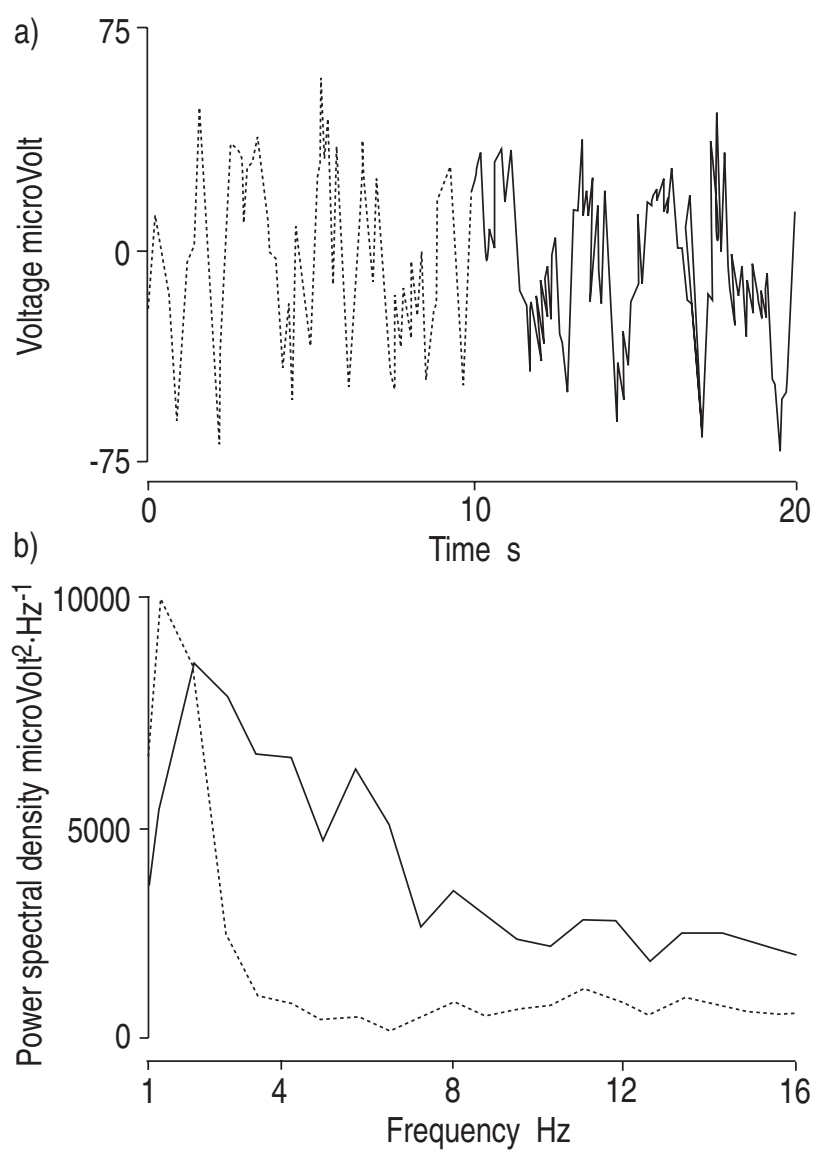

Fig. 1.-Example of the electroencephalographic (EEG) spectral analysis $10 \mathrm{~s}$ before versus $10 \mathrm{~s}$ after the end of the same hypopnoea illustrated on figure 2. a) Twenty second window of the EEG C4A1 tracing around the end of the hypopnoea. Calculation of the power spectral density (PSD) curves before (dotted curve) and after (solid line curve) the end of the hypopnoea. b) Calculation of area under the curves for the frequency bands delta $(1-4 \mathrm{~Hz})$, theta $(4-8 \mathrm{~Hz})$, alpha $(8-12 \mathrm{~Hz})$ and sigma $(12-16 \mathrm{~Hz})$ yield the absolute power values within each frequency band. The normalised spectral power values are the fraction of each band compared to the total power and multiplied by 100 . Comparisons have been carried out between the normalised power values within the same frequency bands of the two PSD curves (i.e. before and after the end) in each manually scored apnoea/hypopnoea.

sleep across the patients, paired t-tests were performed. The Bonferroni correction of $p$-values for multiple comparisons was used and $\mathrm{p} \leqslant 0.01$ was accepted as statistically significant during the paired comparisons.

The frequency band with the most significant power ratio (before versus after end) at apnoea/hypopnoea termination, as evaluated through paired comparisons, was used for the construction of receiveroperator-characteristic (ROC) curves [18]. One ROC curve was constructed for each patient's polysomnogram. The ratio at the end of all apnoeas/hypopnoeas and the ratio around all points which separated two consecutive periods of undisturbed sleep were used for each curve. The area under the ROC curve determined how well the chosen ratio differentiated between apnoea/hypopnoea termination-related changes and undisturbed sleep (the bigger the area, the better the sensitivity).
For the comparison between OSAHS patients and healthy subjects, the mean power of the same frequency band as above was calculated for each polysomnogram using 10-s sliding windows; wake epochs and artefacts were excluded. Three ROC curves were constructed using the mean sleep-related power values of the patients and healthy controls: one for the total sleep time (TST), one for NREM sleep and one for REM sleep; each subject contributed one point to each curve. The area under the ROC curves determined how well the chosen frequency band power differentiated between OSAHS patients and healthy controls.

Independent t-test evaluated the differences between patients and healthy subjects in the band power found to be significant at the end of apnoeas/ hypopnoeas.

One-tailed Pearson's correlation analysis evaluated the relationship between the apnoea/hypopnoea index (AHI) and the mean significant nocturnal band power as well as between AHI and time spent in slow-wave sleep (SWS) and REM sleep across the patients.

All comparisons and ROC curves were based on measurements within 10 -s periods. A p-value $<0.05$ was accepted as statistically significant. Mean values and the SEM or median and interquartile ranges (IQR) are quoted.

\section{Results}

All patients showed a disturbed sleep pattern. Their mean sleep efficiency was $88 \pm 3 \%$ of the sleep period time. Mean time spent in sleep stages 1 and 2 (NREM 1 and 2 ) was $64 \pm 4 \%$ (range: $51-86 \%$ ), in SWS $21 \pm 2 \%$ (range: $1-32 \%$ ) and in REM 15 $\pm 2 \%$ (range: $0.6-25 \%$ ) of the TST. The healthy subjects' mean sleep efficiency was $92 \pm 3 \%$, mean time spent in NREM 1 and 2 was $62 \pm 4 \%$ (range: $49-75 \%$ ), in SWS $21 \pm 3 \%$ (range: 9-29\%) and in REM 17 $\pm 3 \%$ (range: 4-24\%).

Visual scoring of the patients' polysomnograms resulted in a mean AHI of $29 \cdot \mathrm{h}^{-1}$ slept (range: $10-75 \cdot \mathrm{h}^{-1}$ ) and a mean RAI of $23 \cdot \mathrm{h}^{-1}$ (range: $9-52 \cdot \mathrm{h}^{-1}$ slept). Twenty one per cent of all apnoeas and hypopnoeas were terminated without a visually detectable EEG change. Sixty-nine per cent of apnoeas/hypopnoeas were terminated by ASDA arousals, $4 \%$ by $1-3 \mathrm{~s}$ ASDA microarousals and $6 \%$ by awakenings. In NREM sleep $79 \%$ of all apnoeas/hypopnoeas caused visible arousals, $21 \%$ did not. In REM sleep $61 \%$ caused arousals, 39\% did not. In three patients, no apnoeas/hypopnoeas occurred during REM sleep and they were excluded from REM-related comparisons.

The highest intrarater variability was $3 \pm 0.4 \%$ for the arousal index. The variability in sleep scoring was $0.8 \pm 0.5 \%$, in AHI $1.7 \pm 0.9 \%$.

The median duration of apnoeas/hypopnoeas was $22 \mathrm{~s}$ (IQR 17-29 s), their median oxygen desaturation was 5\% (IQR 3-7\%).

\section{Across patients beforelafter comparison}

All apnoeas/hypopnoeas, not affected by movement and/or EEG artefacts, have been included in the 


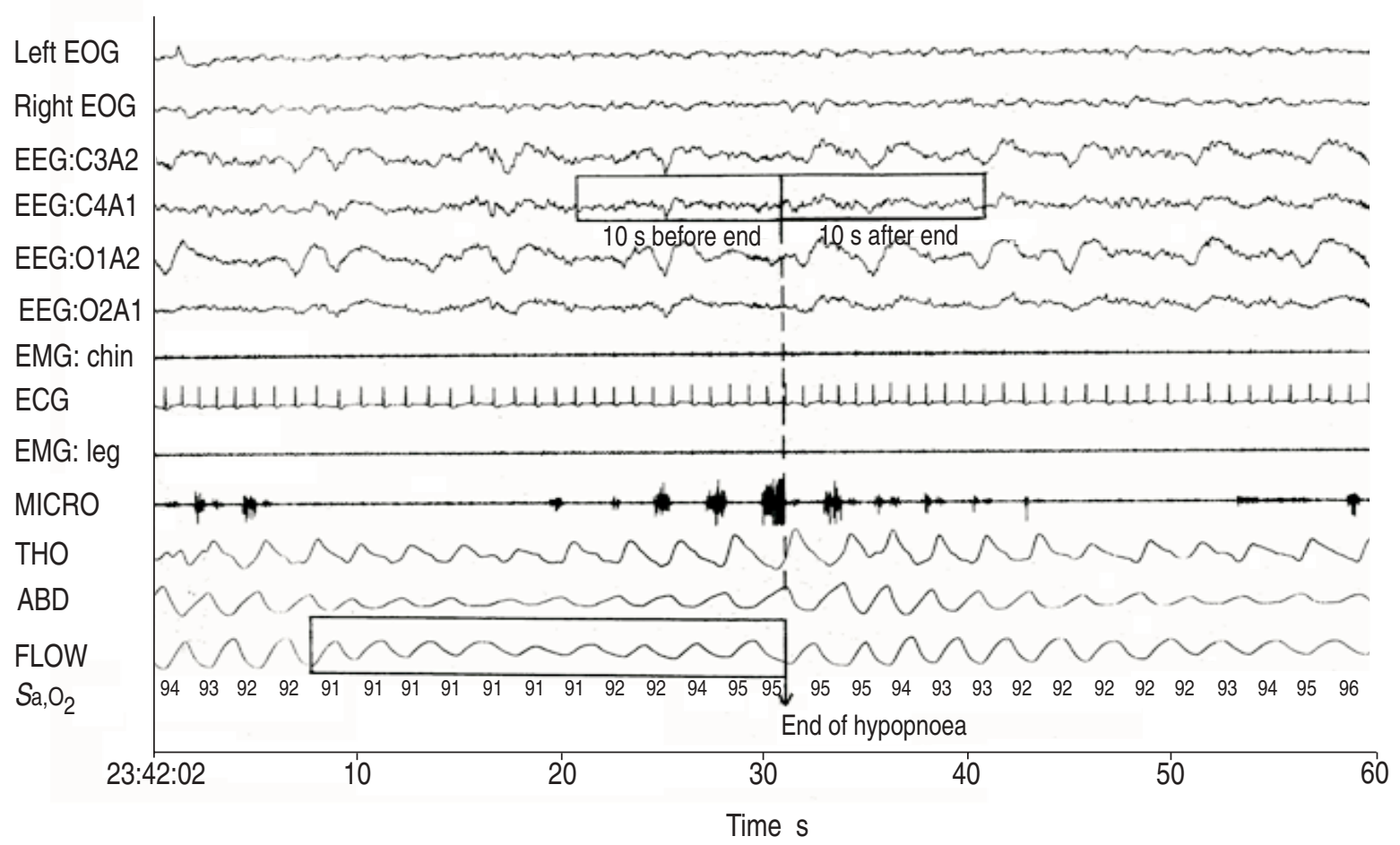

Fig. 2.-Ten second windows chosen for detection of electroencephalographic (EEG) spectral power changes linked to apnoea/hypopnoea termination. The present polysomnographic window illustrates a hypopnoea during nonrapid eye movement sleep, terminated by a visually undetectable manner. EOG: electro-oculogram; EMG: electromyogram; ECG: electrocardiogram; MICRO: microphone; THO: thorax; ABD: abdomen; FLOW: airflow; $S_{\mathrm{a}, \mathrm{O}_{2}}$ : arterial oxygen saturation. End of hypopnoea is indicated by an arrow.

comparisons. In total, 2,596 apnoeas/hypopnoeas and $1,69020 \mathrm{~s}(2 \times 10 \mathrm{~s})$ nonevent periods of undisturbed (baseline) sleep were evaluated across the patients. During NREM sleep 2,395 apnoeas/hypopnoeas versus $1,36320 \mathrm{~s}$ baseline sleep periods were evaluated and during REM sleep 201 apnoeas/hypopnoeas versus 327 baseline periods.
The paired comparison, with each patient contributing one data point to each mean, showed that the postapnoeic/-hypopnoeic decrease in normalised theta power was the most significant $(\mathrm{p}<0.003)$ and consistent change during NREM and REM sleep, in arousal terminating and nonarousal apnoeas/ hypopnoeas (tables 1 and 2). In addition, during

Table 1. - Frequency band power expressed as a fraction of total power multiplied by 100, before and after the end of each apnoea/hypopnoea and during undisturbed sleep, free of apnoeas/hypopnoeas (baseline)

\begin{tabular}{|c|c|c|c|c|c|c|c|c|}
\hline & \multicolumn{2}{|c|}{ All $\mathrm{A}+\mathrm{H}$} & \multicolumn{2}{|c|}{$\mathrm{A}+\mathrm{H}$ and arousal } & \multicolumn{2}{|c|}{$\mathrm{A}+\mathrm{H}$ no arousal } & \multicolumn{2}{|c|}{ Baseline } \\
\hline & Before & After & Before & After & Before & After & Before & After \\
\hline \multicolumn{9}{|l|}{ All } \\
\hline Delta & $41 \pm 0.006$ & $43 \pm 0.01$ & $41 \pm 0.01$ & $42 \pm 0.01$ & $41 \pm 0.01$ & $44 \pm 0.02$ & $42 \pm 0.01$ & $42 \pm 0.01$ \\
\hline Theta & $28 \pm 0.003$ & $24 \pm 0.006$ & $28 \pm 0.005$ & $24 \pm 0.008$ & $28 \pm 0.004$ & $25 \pm 0.008$ & $28 \pm 0.004$ & $28 \pm 0.004$ \\
\hline Alpha & $18 \pm 0.003$ & $19 \pm 0.005$ & $18 \pm 0.005$ & $20 \pm 0.006$ & $18 \pm 0.005$ & $18 \pm 0.008$ & $18 \pm 0.01$ & $18 \pm 0.01$ \\
\hline Sigma & $13 \pm 0.002$ & $14 \pm 0.004$ & $13 \pm 0.04$ & $14 \pm 0.005$ & $13 \pm 0.004$ & $13 \pm 0.008$ & $12 \pm 0.004$ & $12 \pm 0.004$ \\
\hline \multicolumn{9}{|l|}{ NREM } \\
\hline Delta & $41 \pm 0.006$ & $45 \pm 0.01$ & $42 \pm 0.01$ & $41 \pm 0.01$ & $41 \pm 0.01$ & $48 \pm 0.02$ & $45 \pm 0.01$ & $45 \pm 0.01$ \\
\hline Theta & $28 \pm 0.003$ & $24 \pm 0.006$ & $28 \pm 0.004$ & $23 \pm 0.009$ & $28 \pm 0.004$ & $23 \pm 0.009$ & $26 \pm 0.004$ & $26 \pm 0.004$ \\
\hline Alpha & $18 \pm 0.004$ & $18 \pm 0.007$ & $17 \pm 0.006$ & $21 \pm 0.008$ & $18 \pm 0.007$ & $17 \pm 0.008$ & $17 \pm 0.01$ & $16 \pm 0.01$ \\
\hline Sigma & $13 \pm 0.002$ & $13 \pm 0.005$ & $13 \pm 0.003$ & $15 \pm 0.006$ & $13 \pm 0.004$ & $12 \pm 0.007$ & $12 \pm 0.004$ & $11 \pm 0.004$ \\
\hline \multicolumn{9}{|l|}{ REM } \\
\hline Delta & $40 \pm 0.01$ & $41 \pm 0.02$ & $40 \pm 0.01$ & $43 \pm 0.03$ & $40 \pm 0.02$ & $38 \pm 0.02$ & $38 \pm 0.01$ & $38 \pm 0.01$ \\
\hline Theta & $28 \pm 0.006$ & $24 \pm 0.01$ & $28 \pm 0.009$ & $22 \pm 0.01$ & $28 \pm 0.007$ & $26 \pm 0.02$ & $28 \pm 0.01$ & $28 \pm 0.004$ \\
\hline Alpha & $19 \pm 0.005$ & $21 \pm 0.007$ & $19 \pm 0.008$ & $21 \pm 0.01$ & $20 \pm 0.005$ & $21 \pm 0.01$ & $21 \pm 0.01$ & $21 \pm 0.01$ \\
\hline Sigma & $13 \pm 0.006$ & $14 \pm 0.008$ & $13 \pm 0.007$ & $14 \pm 0.008$ & $12 \pm 0.009$ & $15 \pm 0.01$ & $13 \pm 0.01$ & $13 \pm 0.001$ \\
\hline
\end{tabular}

Data are presented as mean \pm SEM. Each patient contributed one data point to each mean; A: apnoea; H: hypopnoea; NREM: nonrapid eye movement; REM: rapid eye movement. 
Table 2. - The p-values, assessed through paired t-tests across the patients, derived from the comparisons between the mean normalised power values within the same frequency bands $10 \mathrm{~s}$ before versus after the end of apnoeas/hypopnoeas as well as during undisturbed, apnoea-/hypopnoea-free (baseline) sleep

\begin{tabular}{|c|c|c|c|c|}
\hline & \multicolumn{3}{|c|}{ Apnoeas/hypopnoeas } & \multirow{2}{*}{$\begin{array}{c}\text { Baseline } \\
\text { sleep }\end{array}$} \\
\hline & All & And arousal & No arousal & \\
\hline $\mathrm{All}^{\#}$ & 2596 & 2001 & 595 & 1690 \\
\hline Delta $_{\mathrm{n}}$ & 0.7 & 0.5 & 0.005 & 0.7 \\
\hline Theta $_{n}$ & $<0.001$ & $<0.001$ & 0.001 & 0.3 \\
\hline Alpha $_{n}$ & 0.008 & 0.001 & 0.1 & 0.5 \\
\hline Sigma $_{n}$ & 0.05 & 0.01 & 0.5 & 0.6 \\
\hline NREM ${ }^{\#}$ & 2395 & 1887 & 508 & 1363 \\
\hline Delta $_{n}$ & 0.02 & 0.5 & 0.002 & 0.3 \\
\hline Theta $_{n}$ & $<0.001$ & $<0.001$ & 0.001 & 0.9 \\
\hline Alpha $_{n}$ & 0.1 & 0.001 & 0.09 & 0.6 \\
\hline Sigma $_{n}$ & 0.5 & 0.01 & 0.1 & 0.4 \\
\hline $\mathrm{REM}^{\#}$ & 201 & 114 & 87 & 327 \\
\hline Delta $_{n}$ & 0.8 & 0.3 & 0.2 & 0.7 \\
\hline Theta $_{n}$ & 0.003 & 0.004 & 0.2 & 0.2 \\
\hline Alpha $_{n}$ & 0.1 & 0.5 & 0.1 & 0.6 \\
\hline Sigma $_{n}$ & 0.1 & 0.5 & 0.1 & 0.9 \\
\hline
\end{tabular}

Each patient contributed one data point to each mean. During the whole night (All) and during nonrapid eye movement (NREM) sleep comparisons were across 15 patients, during rapid eye movement (REM) sleep comparisons were across 12 patients. \#: the number of apnoeas/ hypopnoeas studied; n: the normalised power value of the delta, theta, alpha and sigma frequency bands.

NREM sleep alpha and sigma power increased significantly $(\mathrm{p} \leqslant 0.01)$ in arousal-terminating apnoeas/ hypopnoeas, whereas in nonarousal apnoeas/hypopnoeas there was a significant increase in delta power $(p \leqslant 0.005)$. During REM sleep, no significant power changes were found in nonarousal apnoeas/hypopnoeas.

During periods of undisturbed sleep no significant power changes $(p \geqslant 0.2)$ were found across the patients (table 2).

The mean normalised theta power correlated well with the patients' AHI (rho=0.6, $\mathrm{p}=0.008$ ). AHI and time spent in SWS and in REM sleep were well reciprocally correlated (rho=-0.6, $\mathrm{p}=0.01$; fig. 3 ).

To assess how well theta power fluctuations can differentiate undisturbed sleep from apnoea-/hypopnoea-related EEG changes, one ROC curve was constructed for each patient. Each curve consisted of the theta $a_{\text {normalised }}$ power ratios (theta before_end: $_{\text {ind }}$ theta $\left.{ }_{\text {after_end }}\right)$ at the end of all apnoeas/hypopnoeas and around the point separating all the consecutive $10 \mathrm{~s}$ periods of undisturbed sleep used in the intraindividual comparisons. The areas under the ROC curves, determining the sensitivity of the method, varied between $0.6-0.9$ across the 15 polysomnograms (table 3).

Obstructive sleep apnoealhypopnoea syndrome patients versus healthy subjects

Mean normalised theta power across patients was $25 \pm 0.5$ normalised units (n.u.) and significantly lower than in healthy subjects $28 \pm 1$ n.u. $(F=2.4, p=0.03)$. In patients, mean delta power was $46 \pm 0.1$ n.u., alpha power $17 \pm 0.05$ n.u. and sigma power $12 \pm 0.02$ n.u. In the healthy controls, mean delta power was $44 \pm$ 0.1 n.u., alpha power $17 \pm 0.1$ n.u. and sigma power $11 \pm 0.1$ n.u.; none of the three bands differed significantly between the two groups $(\mathrm{p}>0.2)$.

To assess how well theta power fluctuations can differentiate OSAHS patients from healthy controls, ROC curves were constructed. During NREM sleep the area under the curve was $0.76 \pm 0.1(\mathrm{p}=0.05)$, during REM sleep $0.82 \pm 0.09(\mathrm{p}=0.02)$ and $0.76 \pm 0.1(\mathrm{p}=0.05)$ during the TST (fig. 4).

\section{Discussion}

The present study confirms the hypothesis that the vast majority of apnoeas and hypopnoeas which are not terminated by visually scored arousals are associated with significant spectral power changes. Thus visual arousal scoring in OSAHS patients is less sensitive in detecting all cortical arousal-related changes. To the authors' knowledge this is the first study which evaluates EEG spectral power changes unselected, during REM and NREM sleep, around the end of all apnoeas/hypopnoeas across patients. The present study provides important information on cortical activity changes linked to arousal and nonarousal apnoeas/hypopnoeas during REM and NREM sleep.

\section{Spectral power, sleep states and arousal}

The most significant and consistent change across the 15 patients was the decrease in normalised theta power at the end of apnoeas/hypopnoeas. During NREM sleep when arousals were visible, the decrease in normalised theta derived from the increase in alpha and sigma power. When arousals were not visible, the theta fraction decreased as a result of delta power increase. Across the 12 patients, REM-related arousal terminating apnoeas/hypopnoeas were associated with decrease in theta power fraction alone, which indicates a predominant decrease in the absolute theta power. The 87 REM-related apnoeas/hypopnoeas which were not terminated by visible arousals, were not associated with any significant spectral power changes. This may be due to the limited number of these events across the 12 patients (mean: 6, range: $1-21$ per patient). Furthermore, it suggests that during REM sleep, apnoea/ hypopnoea termination evolves subcortical regions but does not always evoke a cortical reaction.

The mean theta power fraction was significantly lower in the patient group compared to the healthy subjects as a result of the overall increase in sigma and delta power and despite the similar time the two groups spent in NREM 1 and 2, SWS and REM sleep. As the subjects were matched for age and BMI, the findings suggest that the differences in theta power fraction between the two groups are related to the postapnoeic/-hypopnoeic power decrease and the recurrent visible and nonvisible respiratory arousals. 

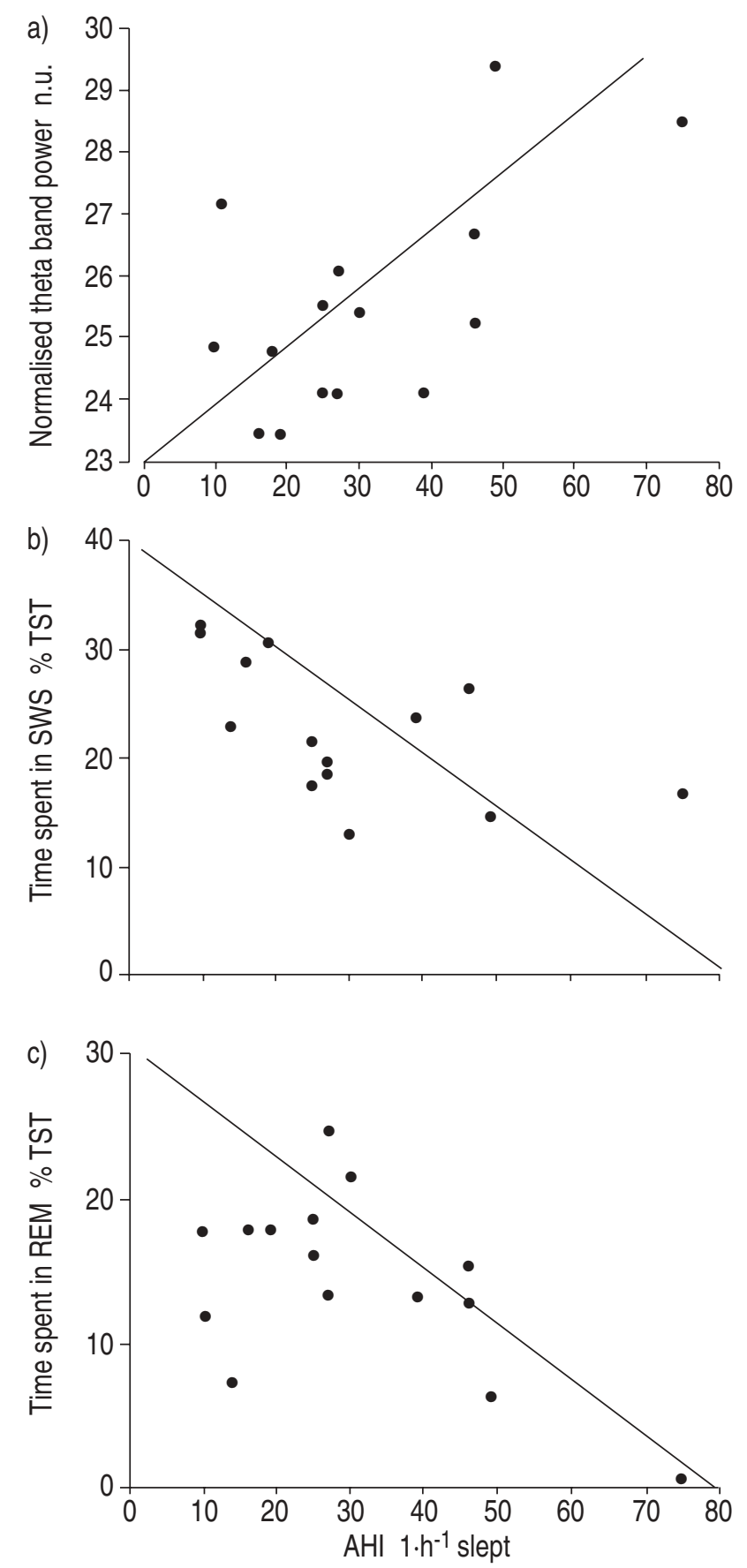

Fig. 3.-a) Good correlation between apnoea-hypopnoea-index (AHI) and mean normalised theta band power ( $\mathrm{rho}=0.6, \mathrm{p}=0.008$ ) across the 15 patients. Good reciprocal correlation between AHI and time spent in b) slow-wave sleep (SWS) as well as in c) rapid eye movement (REM) sleep (rho=-0.6, p=0.01) across the 15 patients. TST: total sleep time; n.u.: normalised units.

These frequent fluctuations of spectral power may contribute to the patients' daytime symptoms. To assess the significance of this assumption, comparisons between objective daytime measurements (such as MSLT) and nocturnal EEG power spectrum would be necessary.

The present findings are consistent with the
Table 3. - Areas under the Receiver-Operator-Characteristic (ROC) curves for each patient based on the theta $a_{\text {normalised }}$ power ratio (theta $a_{\text {before_end: }}: t_{\text {theta }}$ after_end $\left(\operatorname{tn}_{\mathrm{b}}: \mathrm{tn}_{\mathrm{a}}\right)$ ) within $20 \mathrm{~s}$ windows (10 s before versus $10 \mathrm{~s}$ after a point marking either the end of an event or separating a period of undisturbed, baseline sleep). The aim was to assess the accuracy of theta band power in the differentiation of apnoea/hypopnoea termination-related electroencephalographic changes from baseline sleep

\begin{tabular}{lcccc}
\hline $\begin{array}{l}\text { Patient } \\
\text { no. }\end{array}$ & $\begin{array}{c}\mathrm{AHI} \\
\mathrm{h}^{-1}\end{array}$ & $\begin{array}{c}\mathrm{tn}_{\mathrm{b}}: \mathrm{tn}_{\mathrm{a}} \\
(\mathrm{SEM} ; \mathrm{p}-\mathrm{value})\end{array}$ & $\begin{array}{c}\mathrm{RE} \\
\mathrm{no} .\end{array}$ & $\mathrm{BL}$ no. \\
\hline 1 & 75 & $0.7(0.04 ;<0.001)$ & 256 & 40 \\
2 & 49 & $0.7(0.05 ; 0.002)$ & 226 & 32 \\
3 & 39 & $0.7(0.03 ;<0.001)$ & 249 & 92 \\
4 & 46 & $0.8(0.03 ;<0.001)$ & 276 & 20 \\
5 & 46 & $0.7(0.02 ;<0.001)$ & 293 & 218 \\
6 & 25 & $0.9(0.01 ;<0.001)$ & 179 & 168 \\
7 & 25 & $0.7(0.03 ;<0.001)$ & 188 & 120 \\
8 & 27 & $0.6(0.03 ;<0.001)$ & 160 & 148 \\
9 & 27 & $0.9(0.02 ;<0.001)$ & 149 & 173 \\
10 & 30 & $0.6(0.03 ; 0.004)$ & 198 & 100 \\
11 & 10 & $0.9(0.03 ;<0.001)$ & 71 & 32 \\
12 & 10 & $0.7(0.05 ; 0.002)$ & 49 & 134 \\
13 & 14 & $0.7(0.03 ;<0.001)$ & 92 & 109 \\
14 & 16 & $0.7(0.03 ;<0.001)$ & 105 & 131 \\
15 & 19 & $0.7(0.03 ;<0.001)$ & 105 & 173 \\
Mean & 29 & 0.7 & 173 & 113 \\
Range & $10-75$ & $0.6-0.9$ & $49-293$ & $20-218$ \\
\hline
\end{tabular}

RE: respiratory events (apnoeas/hypopnoeas); BL: number of baseline sleep periods; SEM: standard error of the mean; AHI: apnoea/hypopnoea index.

outcomes of Svanborg et al. [9] who analysed the EEG power spectrum in the course of apnoeic/ hypopnoeic episodes based on 2-s epochs and found

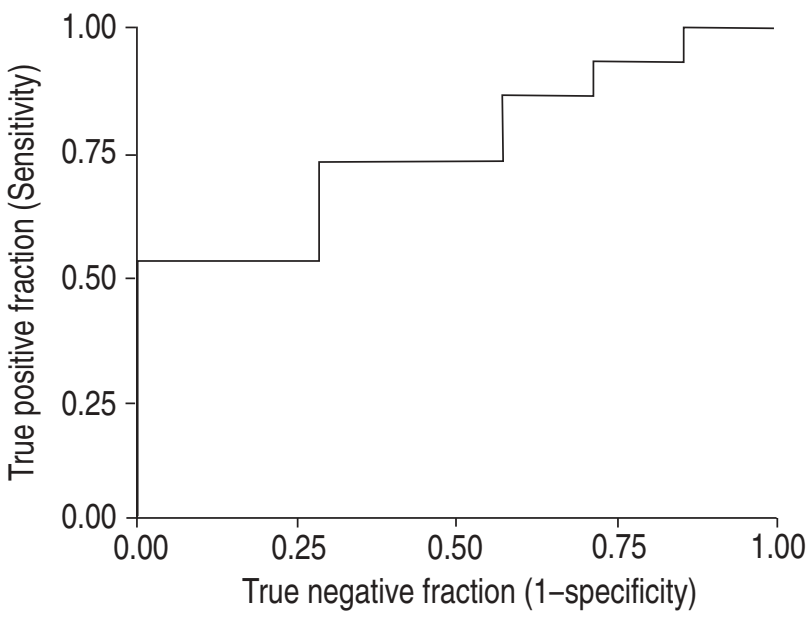

Fig. 4. - The Receiver-Operator-Characteristic (ROC) curve assesses how well the mean normalised theta power differentiates between obstructive sleep apnoea/hypopnoea syndrome (OSAHS) patients and healthy controls. The calculation of the mean theta power is based on spectral analysis within $10 \mathrm{~s}$ sliding windows across the whole night's electroencephalography. Each subject contributes one point to each mean; i.e. the ROC curve consists of 22 points (15 patients and seven healthy controls). The area under the curve is 0.76 . With 1.0 being the highest possible area, mean theta power is a good marker for the differentiation between OSAHS patients and healthy subjects. 
an increase in delta power towards the end of apnoeas/ hypopnoeas and a decrease at their termination as faster activities became more prominent. BERRY et al. [6] identified a cyclical delta power increase in sleep apnoeics, parallel to increased inspiratory effort. The EEG spectral analysis was performed using sequential $1 \mathrm{~s}$ windows. Both studies identified differences between NREM and REM sleep, as no significant changes in delta band power were identified during REM-related apnoeas/hypopnoeas. BLACK et al. [19] analysed the EEG power spectrum around the peaks of oesophageal pressure (Poes) using 4-s windows, during NREM 2 in 15 upper airway resistance syndrome patients, and similarly identified a progressive increase in delta power preceding those peaks. An increase in the power of higher frequencies was identified at $P_{\text {oes }}$ reversal. Although changes were more significant at arousal terminating "events", they were also present when arousals were not visible. The present study focused around the end of apnoeas/ hypopnoeas and did not look into EEG power changes in the evolution of these events. Therefore, the study could not confirm the previously found significant delta power increase towards the end of apnoeas/hypopnoeas, identified through consecutive comparisons of short intervals prior to and during apnoeas/hypopnoeas. The aim of the present study was to improve detection of EEG changes at the end of apnoeas/hypopnoeas. Using longer windows, the analysis looked into changes in delta, theta, alpha and sigma power and their interaction and delivered 10-s based power values. The most consistent finding across the 2,596 events studied, is the theta power decrease. In the majority of NREM apnoeas/hypopnoeas this resulted from the increase in higher frequencies, which made the EEG changes visually detectable. The rise in high activity is consistent with the findings of Svanborg and Guilleminault [9] and BLACK et al. [19]. Furthermore, the present study found that events with nonvisible EEG changes and no alpha/sigma increase induced a significant delta increase. Hence, this rise in slow activity in response to apnoeas/hypopnoeas represents, as previously suggested [9], a form of arousal. DeCARLi et al. [20] support the postevent alpha and/or theta increase but not delta alone, based on the outcomes of wavelet transform, a different technique of EEG analysis.

The present study confirms the previously found power differences between REM- and NREM-related apnoeas/hypopnoeas: visible changes in REM sleep were associated with significant theta decrease alone, nonvisible changes showed no significant power changes; hence, no changes in delta power have been detected $[6,9]$. The present findings provide information on differences between arousal and nonarousal REM- and NREM-related apnoeas/hypopnoeas. Previous studies did not look at these differences either because only NREM-related apnoeas/hypopnoeas were studied [19] or because differences in respect to the visibility of arousals were not evaluated [9].

The increased EMG activity during visible cortical arousals could potentially alter the EEG power spectrum and induce differences between visible versus nonvisible arousals. Muscle artefacts (movement and breathing) would influence alpha, sigma and low delta $(0.2-2.2 \mathrm{~Hz})$ but not theta frequency band power, as BRUNNER et al. have demonstrated [21]. However, the importance of this factor would definitely be in certainty evaluated through the elimination of EMG influences from the EEG signal, which was not performed during the present study.

\section{Sleep quality}

Sleep quality was not significantly different between patients and healthy controls. This was due to: 1) the impaired sleep quality of healthy subjects under in-lab conditions; and 2) the inconsistency among patients as to their disease severity ranging from mild, moderate to severe, yielding differences in their sleep quality. The latter is supported by the good reciprocal correlation found between AHI and time spent in SWS and REM sleep and the good correlation between AHI and theta power across the patients. The differences in sleep quality between patients and healthy controls are therefore better preserved through the range of time spent in each sleep state.

\section{Study limitations}

Limitations of this study include definitions, the use of thermistor for airflow detection, the unequal number between apnoeas/hypopnoeas and periods of undisturbed sleep used in the comparisons, as well as the number of subjects studied. A further limitation may be the use of only two bins: NREM and REM sleep.

There are many definitions of hypopnoeas and arousals used in the literature. The hypopnoea definition presently used, fulfils the ASDA recommendations [11]. The inclusion of arousals or desaturation in the definition may lead to a greater association with arousals than would be found using other ASDA accepted definitions, i.e. based on thoraco-abdominal movement or nasal pressure alone. The arousal definition used, has been used in previous studies and validated against respiratory events and outcomes [4]. The thermistor is a widely used good qualitative detector of airflow detection. It is unlikely that the limited accuracy of this sensor may have altered the outcomes of the EEG spectral analysis. This is supported by the fact that the findings are in keeping with the outcomes of BLACK et al. [19], who used oesophageal manomentry to monitor changes in respiratory effort.

All the 2,596 apnoeas/hypopnoeas and 1,690 undisturbed sleep periods, which were not affected by artefacts, across the 15 polysomnograms were analysed. This represents a large body of data with which to answer the questions posed. The present results are not only robust but represent the largest body of events which have been so analysed. The study is thus adequately powered to detect clinically important differences.

The two bins, NREM and REM sleep, were selected as a result of their different characteristics: REM sleep 
is a low voltage, high frequent state; its baseline, nonevent, power spectrum is different from the NREM spectrum. However, it was beyond this paper's aims to look into apnoea/hypopnoea-related differences across the four different NREM sleep stages.

In conclusion, the present study has shown that novel techniques may improve and simplify detection of electrographical changes at apnoea/hypopnoea termination. The most consistent significant change is the decrease in theta power, which during NREM sleep is either associated with an increase in high frequencies (alpha, sigma) or delta increase.

The detection of theta power changes is, as the ROC curves demonstrate, a sensitive method to pick up apnoea-/hypopnoea-related EEG changes and also to differentiate between OSAHS patients and healthy subjects. Since part of the REM-related apnoeas/ hypopnoeas is not associated with significant theta power changes, the proposed method may only be used as part of a tool for improved and simplified diagnosis of OSAHS.

Further validation, estimation of a cut-off value for the differentiation between patients and nonpatients and correlation analysis between this electroencephalographic activity marker and sleepiness severity as well as cognitive function impairment, are necessary.

Acknowledgements. The authors would like to thank the European Respiratory Society for its financial support, N. McArdle for helpful comments on the manuscript and E. Dolan for secretarial assistance.

\section{References}

1. Kingshott RN, Engleman HM, Deary IJ, Douglas NJ. Does arousal frequency predict daytime function? Eur Respir J 1998; 12: 1264-1270.

2. Cheshire K, Engleman HM, Deary IJ, Shapiro C, Douglas NJ. Factors impairing daytime performance in patients with sleep apnea/hypopnea syndrome. Arch Intern Med 1992; 152: 538-541.

3. Guilleminault C, Partinen M, Quera-Salva MA, Hayes B, Dement WC, Nino-Murcia G. Determinants of daytime sleepiness in obstructive sleep apnea. Chest 1988; 94: 32-37.

4. Rees K, Spence DPS, Earis JE, Carverley PMA. Arousal responses from apneic events during NREM sleep. Am J Respir Crit Care Med 1995; 152: 10161021.

5. Martin SE, Wraith PK, Deary IJ, Douglas NJ. The effect of non-visible sleep fragmentation on daytime function. Am J Respir Crit Care Med 1997; 155: 15961601.

6. Berry RB, Asyali MA, McNellis MI, Khoo MCK. Within-night variation in respiratory effort preceding apnea termination and EEG delta power in sleep apnea. J Appl Physiology 1998; 85: 1434-1441.

7. Stradling JR, Davies RJ. Is it necessary to record sleep? Sleep 1996; 19: S251-S254.

8. Pardey J, Roberts S, Tarassenko L, Stradling J. A new approach to the analysis of the human sleep/wakefulness continuum. J Sleep Res 1996; 5: 201-210.

9. Svanborg E, Guilleminault C. EEG Frequency changes during sleep apneas. Sleep 1996; 19: 248-254.

10. Rechtschaffen A, Kales A. A manual of standardised terminology: techniques and scoring systems for sleep stages of human subjects. Publication No. 204. Washington, National Institutes of Health, 1968.

11. ASDA Task Force Report. EEG Arousals: Scoring rules and examples. Sleep 1992; 15: 173-184.

12. American Academy of Sleep Medicine Task Force Report. Sleep-related breathing disorders in adults: recommendations for syndrome definition and measurement techniques in clinical research. Sleep 1999; 22: 667-689.

13. Tompkins WJ. Biomedical Digital Signal Processing. Englewood Cliffs, New Jersey, Prentice Hall, 1993; pp. 184-192; pp. 216-236.

14. Papoulis A. Signal Analysis. 2nd ed. London, McGraw-Hill International Incorporation, 1985; pp. 101-173; pp. 300-386.

15. Welch PD. The use of fast Fourier transformation for the estimation of power spectra: A method based on time averaging over short modified periodograms. IEEE Trans Audio Electroacoust 1967; AU-15: 70-73.

16. Oppenheim AV, Willsky AV, Young IT. Signals and systems. London, Prentice Hall International Incorporation, 1983; pp. 414 422.

17. Mitra SK, Kaiser JF. Handbook for digital signal processing. New York, John Wiley \& Sons, 1993; pp. 175-278.

18. Zweig MH, Campbell G. ROC plots: a fundamental evaluation tool in clinical medicine. Clin Chem 1993; 39: pp. 561-577.

19. Black J, Guilleminault C, Colrain IM, Carrillo O. Upper Airway Resistance Syndrome: central electroencephalographic power and changes in breathing effort. Am J Respir Crit Care Med 2000; 162: 406-411.

20. De Carli F, Nobili L, Gelcich P, Ferrillo F. A method for the automatic detection of arousals during sleep. Sleep 1999; 22: 561-572.

21. Brunner DP, Vasko RC, Detka CS, Monahan JP, Reynolds III CF, Kupfer DJ. Muscle artifacts in the sleep EEG: Automated detection and effect on allnight EEG power spectra. J Sleep Res 1996; 5: 155164. 